\title{
Dispersión urbana y nuevos desafíos para la gobernanza (metropolitana) en América Latina: el caso de Santiago de Chile*
}

\author{
DIRK HEINRICHS**, HENNING NUISSL** Y CLAUDIA RODRÍGUEZ SEEGER*** \\ ** Centro Helmholtz de Investigación Medioambiental (UFZ) \\ *** Pontificia Universidad Católica de Chile
}

\begin{abstract}
Massive expansion of urban areas is not a new experience to Latin American cities. However, the profound social, ecological and economic implications of urban sprawl are only today starting to become a major concern in the continent's big agglomerations. By taking Santiago de Chile as an example this paper discusses the challenges posed to urban governance by urban sprawl. It first summarizes recent trends in residential housing production where large scale residential 'megaprojects' have become highly significant. Second, by looking at these trends from the perspective of the international sprawl literature it finds that urban sprawl in Santiago is largely driven by policy and decision making. It thirdly constructs a basic analysis of sprawl governance in terms of actors, institutions and their interplay on the basis of the few existing studies and the author's own ongoing research. Finally, the paper suggests some entry points and broad strategies to address sprawl.
\end{abstract}

\section{KEY WORDS: urban sprawl, urban expansion, governance, Santiago de Chile}

RESUMEN La expansión acelerada de las áreas urbanas no es algo nuevo para las ciudades latinoamericanas. Sin embargo, es sólo ahora que las profundas implicaciones sociales, ecológicas y económicas de la dispersión urbana empiezan a convertirse en una preocupación fundamental de las grandes aglomeraciones del continente. El presente artículo toma a Santiago de Chile como ejemplo para discutir los desafíos que la dispersión urbana supone para la gobernanza. En primer lugar, resume tendencias recientes en producción de viviendas, donde los "megaproyectos" residenciales a gran escala han sido muy importantes. En segundo lugar, considerando estas tendencias desde la perspectiva de la bibliografía internacional sobre dispersión, concluye que la dispersión urbana en Santiago está ampliamente impulsada por las políticas públicas y la toma de decisiones. En tercer lugar, construye un análisis básico de la gobernanza de la dispersión en términos de actores, instituciones y su interacción, tanto sobre la base de los pocos estudios existentes, como de las propias investigaciones en curso de los autores. Finalmente, el artículo sugiere campos de acción y lineamientos estratégicos de carácter general como puntos de partida para afrontar la dispersión.

PALABRAS CLAVE: dispersión urbana, expansión urbana, gobernanza, Santiago de Chile

Este trabajo está siendo desarrollado como parte de la iniciativa de investigación "Risk Habitat Megacity" (www. risk-habitat-megacity.org) y ha sido posible con la ayuda de la Helmholtz Initiative and Networking Fund. Estamos en deuda con Michael Lukas por sus agudos comentarios y contribuciones efectuados a una versión anterior de este artículo. También damos las gracias a Annegret Kindler por su apoyo en el diseńo y elaboración de los mapas.

Recibido el 25 de agosto de 2008, aprobado el 5 de octubre de 2008.

Correspondencia: Dirk Heinrichs, Henning Nuissl, Departamento de Sociología Urbana y Medioambiental, Permoserstraße 15, 04318 Leipzig, Alemania. E-mail: dirk.heinrichs@ufz.de, henning.nuissl@ufz.de. Claudia Rodríguez Seeger, Facultad de Arquitectura, Diseño y Estudios Urbanos, El Comendador 1916, Pedro de Valdivia Norte, Casilla 16002 - Correo 9, Santiago de Chile, Chile. E-mail: crodrig@uc.cl. 


\section{Introducción}

La dispersión urbana ${ }^{1}$ está cambiando paisajes a través de toda la región latinoamericana. A la vez, están empezando a hacerse evidentes sus profundas implicaciones sociales, ecológicas y económicas, que se expresan en indicadores como el creciente consumo de tierra per cápita y la disminución de la densidad de población en las áreas urbanas en expansión. Las manifestaciones de este fenómeno suelen estar bien documentadas en la bibliografía y han sido mostradas en varios estudios específicos del caso de Santiago de Chile (ver, p. ej., las contribuciones a De Mattos e Hidalgo, 2007 y Galetovic, 2006; ver también Ducci, 2000; Ducci y González, 2006; Green y Soler, 2005; Romero y Vásquez 2006; Paz Castro, 2006; López, 2006). Asimismo, los estudios en referencia aluden a los cambios en el acceso de las personas a recursos y servicios, nuevos patrones de inclusión/exclusión social, consumo extensivo de suelo en lugares que a veces no están integrados a la ciudad, lo cual muchas veces se asocia con el aumento de la demanda de infraestructura de transporte y la pérdida de los servicios de ecosistemas.

La expansión de las viviendas contribuye de manera significativa a la tendencia descrita. Mientras que tradicionalmente se ha tratado este fenómeno como los efectos de los (cambiantes) patrones de demanda según estrato socioeconómico, en las décadas recientes se hace cada vez más explícito el rol que han tenido los modos de producir viviendas y su evolución hacia desarrollos inmobiliarios de gran escala. En América Latina y en particular en Santiago de Chile, esta tendencia a menudo toma la forma de los así llamados megaproyectos. Ellos no sólo suponen un nuevo tipo de desarrollo urbano (Borsdorf e Hidalgo, 2005a; Hidalgo, Borsdorf y Sánchez, 2007), sino también contribuyen a nuevas formas de morfología urbana y suburbanización.

En nuestro artículo examinamos el caso de megaproyectos residenciales, en un intento de demostrar el régimen de dispersión urbana de Santiago de Chile y de desenmarañar la densa interrelación de actores, instituciones y procesos de toma de decisiones. En primer lugar, el artículo resume las actuales tendencias de dispersión en base a variables demográficas y de uso de suelo urbano, sintetiza las recientes tendencias en producción de viviendas y ofrece un breve resumen de las políticas relacionadas con la dispersión. En segundo lugar, construye un análisis básico de gobernanza en términos de actores, instituciones y su interacción en los procesos de construcción urbana. Elaboramos nuestro análisis sobre la base de los pocos estudios existentes y añadimos las primeras observaciones que emanan de nuestras propias investigaciones. En tercer lugar, el artículo concluye con la identificación de amplios campos de acción y algunos lineamientos estratégicos de carácter general, como puntos de partida para afrontar la dispersión.

\footnotetext{
$1 \quad$ Ver por ejemplo Peiser (2001): “El término 'dispersión urbana’ se usa de varias formas para referirse al consumo desmesurado de suelo, desarrollo monótono ininterrumpido, desarrollo discontinuo a base de saltos ('leapfrog discontinuous development') y el uso ineficiente del suelo.” Este fenómeno es ampliamente conocido en inglés como "urban sprawl". No obstante, tal como lo manifiestan De Lázaro y Torres et al. "no se puede hablar de dispersión urbana o 'urban sprawl' y quizás tampoco de ciudad dispersa, ya que la ciudad y lo urbano por definición son concentrados y densos, y no dispersos, aunque sí podríamos utilizar aquí las palabras hábitat o asentamiento" (De Lázaro y Torres et al. 2006, p. 70). Tratándose, sin embargo, de un hábitat disperso que se extiende en forma centrífuga a partir de un núcleo urbano, en este artículo se adoptará el término de 'dispersión urbana', según la propuesta realizada por Peiser (2001).
} 
Sostenemos que el asunto de la dispersión y sus implicaciones en Santiago pueden no estar siendo tratados de forma adecuada en el nivel de proyectos individuales, pues se requeriría un enfoque regional y un discurso público amplio e informado que incluya al público general. En este contexto regional, un área de investigación sería los potenciales de cooperación intercomunal. Las conclusiones son tentativas y reflejan el trabajo en curso.

\section{Dispersión urbana residencial en Santiago de Chile: tendencias actuales}

Las ciudades en América Latina manifiestan considerables tasas de crecimiento en términos de desarrollo del suelo en la periferia urbana. Mientras que en ciudades como Lima, Bogotá o Rio de Janeiro esta tendencia va acompañada de un aumento de la población, otras ciudades como Santiago de Chile o Buenos Aires experimentan procesos simultáneos de descenso de la población central y suburbanización.

El Área Metropolitana de Santiago de Chile (AMS), con su actual población de unos 5,5 millones de habitantes, es un buen caso para demostrar la evolución de la dispersión urbana y los factores que influyen en este proceso en América Latina. El AMS no es una entidad político-administrativa en sí misma, sino una aglomeración de municipalidades soberanas. En efecto, ella es parte de una Región Metropolitana (RM) mucho más grande, con una población de alrededor de 6,2 millones de habitantes, constituyendo el centro de uno de los países más urbanizados de la región. A menudo Santiago es tomado como ejemplo para explicar el modelo ideal de crecimiento desde la "ciudad compacta" colonial a lo que hoy es conocido como una "ciudad fragmentada" (ver Bähr y Mertins, 1995).

\section{CRECIMIENTO ESPACIAL DE SANTIAGO DE CHILE (1600-2000)}

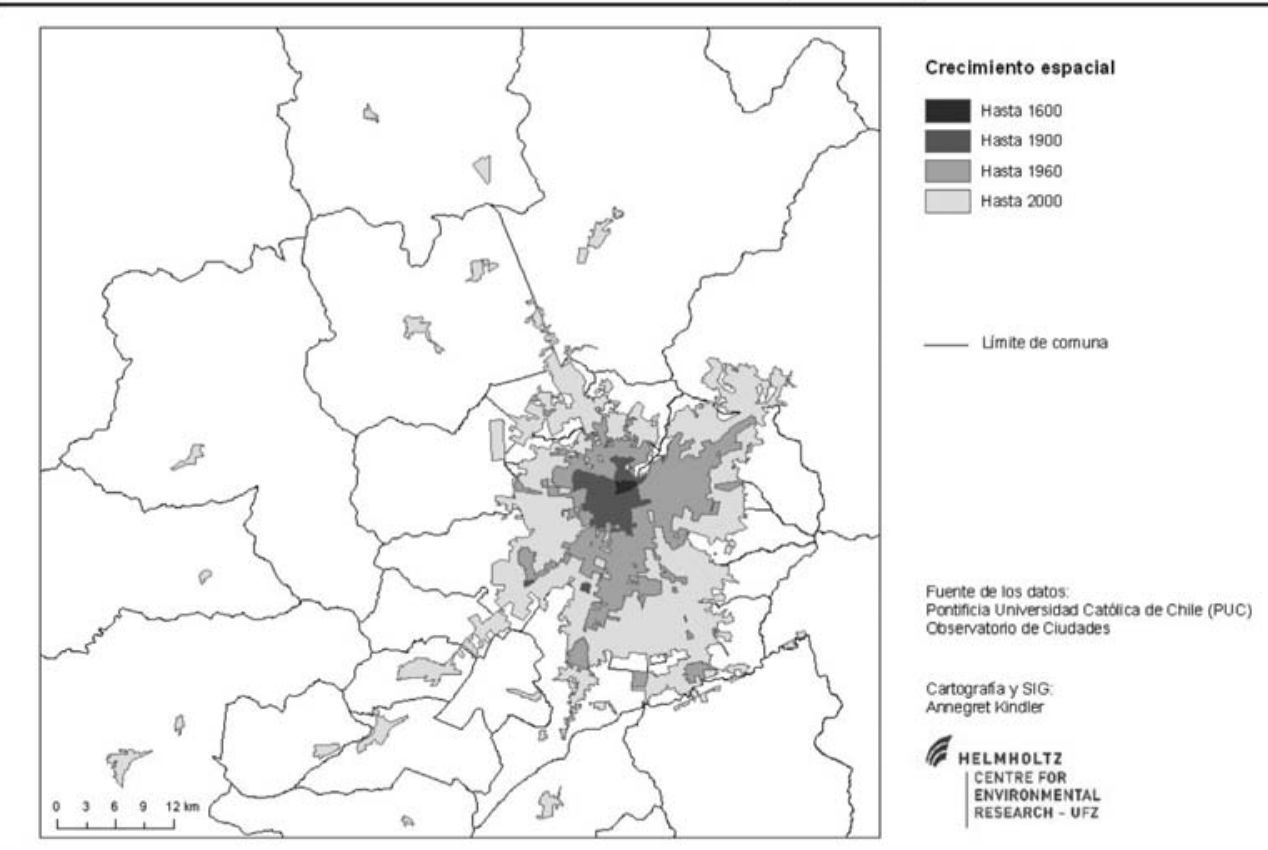

Fuente: Autores, basado en IEUT, 2003 
Junto con la mencionada fragmentación se ha producido una rápida expansión horizontal, la cual ha sido especialmente pronunciada en las décadas recientes, cuando el área urbana construida se duplicó de alrededor de $330 \mathrm{~km}^{2}$ en 1980 a más de $600 \mathrm{~km}^{2}$ en 2004 (Petermann, 2006). Por otro lado, el crecimiento de la población urbana durante el mismo período de tiempo se ralentizó, pasando de más de un 2\% a menos de un 1,5\% (Galetovic y Jordan, 2006). En efecto, la densidad de población disminuyó de 93 a 85 habitantes/há durante dicho período.

En general, una población que se consolida sobre un área física en expansión esconde una movilidad intraurbana muy pronunciada. Entre 1997 y 2002, más de 150.000 personas se mudaron anualmente dentro del AMS (Rodríguez, 2007). La importancia de esta cifra es que ella implica una tendencia consistente de reubicación hacia las comunas periféricas (ver Escolano y Ortiz, 2007).

\section{Mapa 2}

CAMBIO EN EL TAMAÑO DE LA POBLACIÓN POR COMUNA (1992 - 2002) EN EL ÁREA METROPOLITANA DE SANTIAGO DE CHILE (AMS)

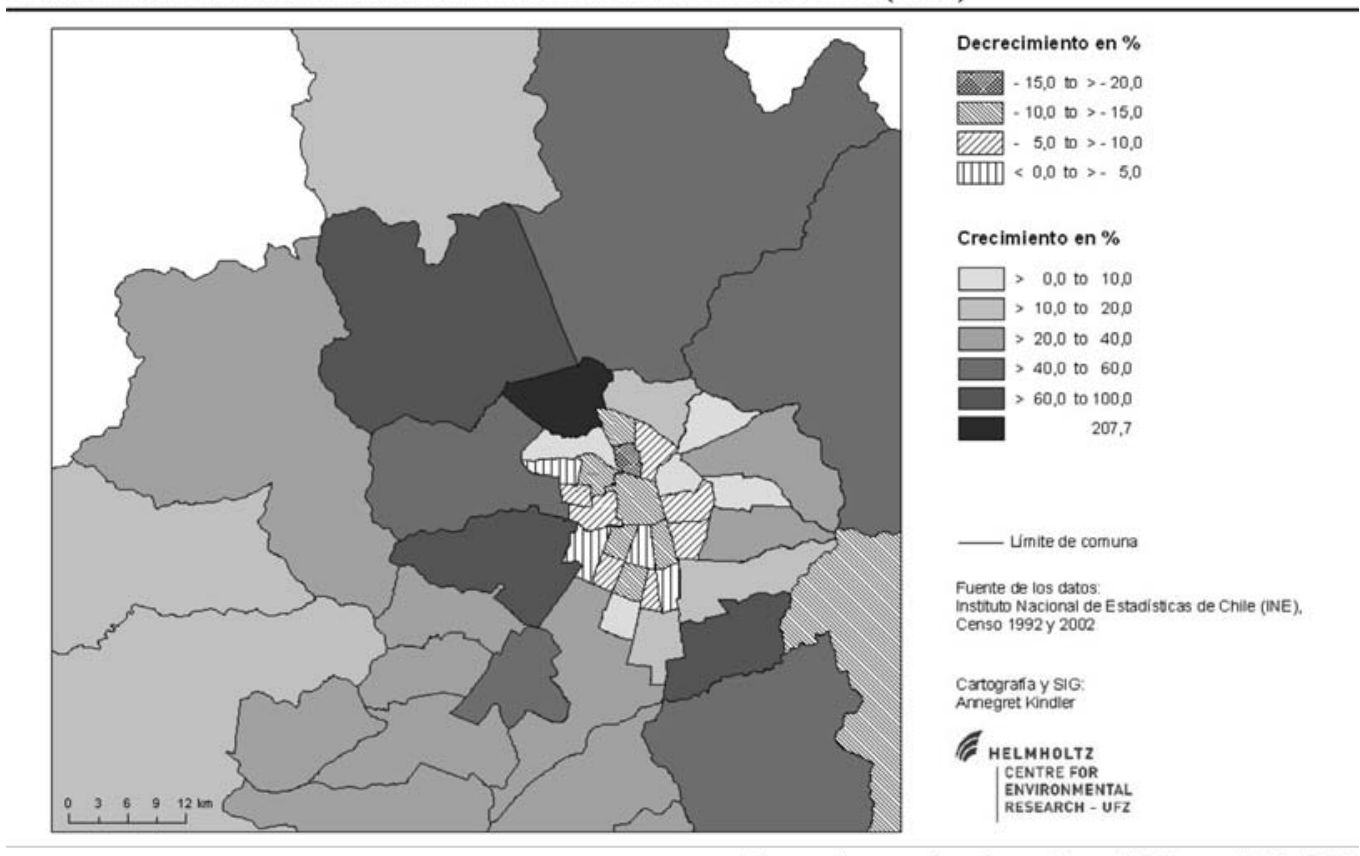

Fuente: Autores, basado en Datos del Censo 1992 y 2002

En conjunto, entre 1992 y 2002, casi todas las comunas centrales y pericentrales han perdido población a un ritmo significativo. En cambio las localidades periféricas, como las comunas de Puente Alto, Maipú, Lampa o Quilicura, tuvieron un repentino aumento de población con tasas de hasta $200 \%$. La última década experimentó el aumento de proyectos residenciales de gran escala, en especial, en la periferia (Borsdorf e Hidalgo, 2005b). Estos megaproyectos, que se estima representan más del $70 \%$ de la producción de viviendas en los últimos años, están en su mayoría diseñados como barrios cerrados para -en algunos casos- más de 50.000 personas. 
Su construcción incluye infraestructura y servicios (como complejos comerciales y escuelas), en algunos casos la autopista que los conecta con el centro de la ciudad, así como obras públicas en general. Estos desarrollos suelen ocurrir en suelo no urbanizado en la periferia urbana y, en forma creciente, cada vez más lejos de ésta. Dos ejemplos son Piedra Roja (65.000 habitantes) y Valle Grande (57.000 habitantes). Esta forma de urbanización ya no está en absoluto reservada a grupos de ingresos elevados, sino que se dirige a un espectro socioeconómico mucho más amplio, que incluye el grupo de ingresos medio-bajos.

Mapa 3

MEGAPROYECTOS Y ZONAS DE DESAROLLO URBANO CONDICIONADO EN EL ÀREA METROPOLITANA DE SANTIAGO DE CHILE (AMS)

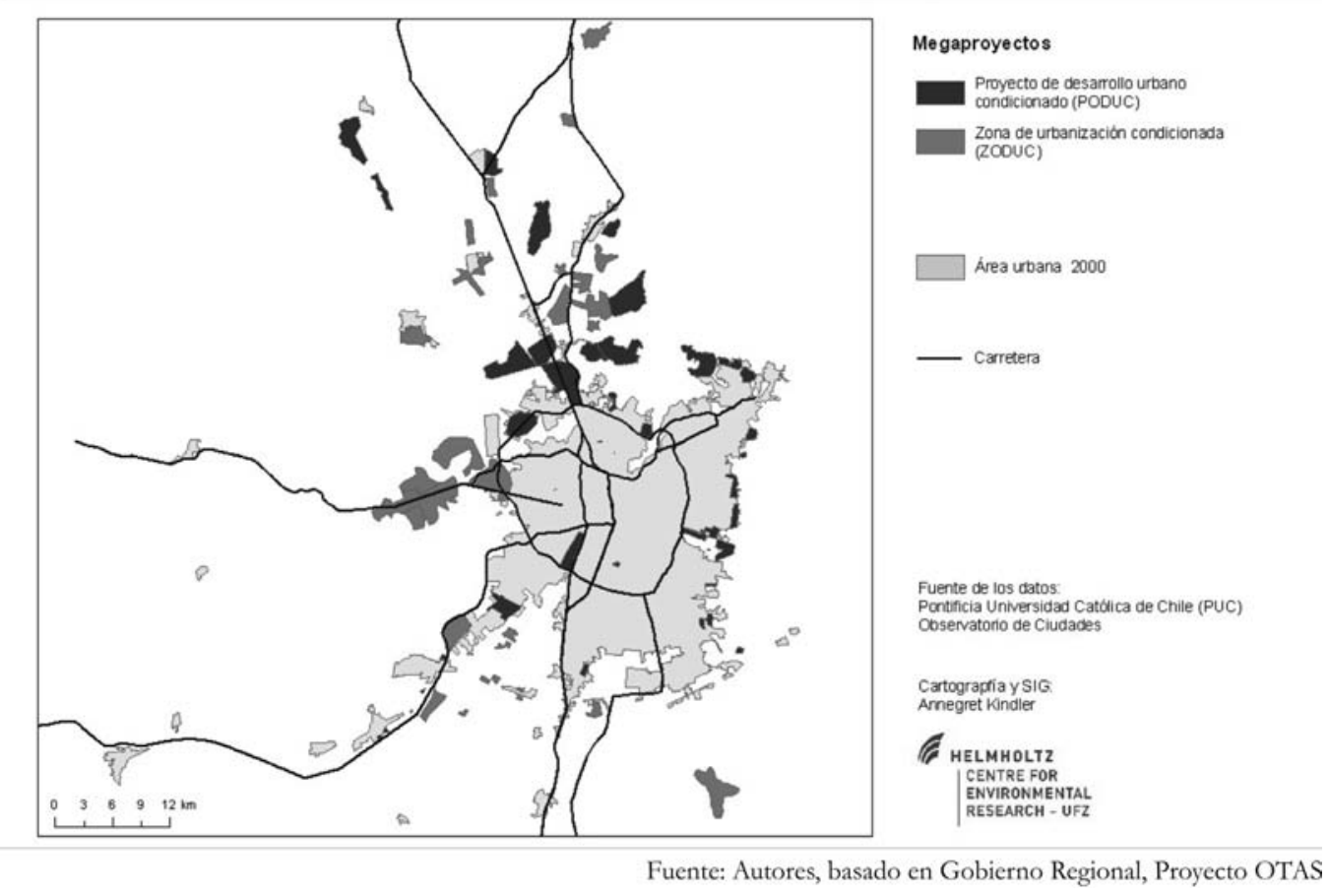

Explicamos el desarrollo de megaproyectos residenciales y la elección de ubicación en la periferia urbana, a través de la intención por parte de los promotores inmobiliarios de capitalizar el potencial de brecha que se genera entre el valor del suelo no urbanizado y el máximo beneficio económico resultante de urbanizarlo con los mayores estándares y rentabilidades de uso. El estudio de Arriagada y Simioni (2001) sobre la dinámica del valor del suelo en Santiago menciona este potencial. Mientras que el precio del suelo aumentó anualmente alrededor de un $22,5 \%$ entre 1992 y 1998 como media comunal, en varias comunas periféricas dicha cifra superó el 100\%. Sin embargo, sólo es posible comprender los enormes retornos económicos del desarrollo inmobiliario en la periferia urbana de Santiago, en un marco particular de gobernanza. Nos ocuparemos de este aspecto en la siguiente sección. 


\section{¿Cómo explicar la dispersión urbana en Santiago?}

La corriente más importante de la bibliografía sobre suburbanización y dispersión urbana atribuye este fenómeno a dos causas diferentes. Una línea de razonamiento se centra en la demanda y sostiene que la dispersión urbana básicamente refleja las preferencias y aspiraciones de (una mayoría de) familias que quieren vivir una vida suburbana. Desde este punto de vista, los desarrollos suburbanos aparecen en gran parte como consecuencia de la acción de planificadores e inversionistas, la cual estaría dirigida a satisfacer dicha demanda. La otra línea de argumentación, igualmente importante, sostiene que el fenómeno de la dispersión urbana en absoluto es sólo el resultado (inevitable) de la demanda de los hogares individuales de más viviendas suburbanas (respaldado por factores económicos y sociales), sino también "el producto de las políticas gubernamentales" (Jackson, 1985). Este argumento se apoya en la afirmación que el desarrollo urbano y el uso del suelo están fuertemente dirigidos por la alianza de intereses privados y las políticas públicas pertinentes. Al buscar las razones para la actual dispersión urbana en Santiago de Chile, encontramos varios indicios que conllevan tanto a una como a otra corriente de argumentación en debate.

En relación con el aspecto que refuerza el argumento de la demanda para la dispersión, algunos autores señalan un ideal de vida suburbana permanente en Chile (también aplicable a otros países de América Latina), el cual se refiere a las preferencias de las élites, que siempre habrían intentado distanciarse de las estrecheces, la aglomeración y la miseria del centro de la ciudad (De Ramón, 2007). En Santiago, este proceso se manifiesta como reubicaciones del antiguo centro urbano hacia las comunas pericentrales de Providencia, Vitacura y Las Condes. Sólo últimamente los residentes de ingresos elevados amplían los límites urbanos, construyendo nuevas viviendas lujosas en la periferia urbana e incluso más allá de ésta. Esta orientación suburbana, ayudada por el intento de modernizar la ciudad e invertir en infraestructura para hacer que la capital chilena sea competitiva a escala global (De Mattos, 2005), ha sido ampliamente adoptada por la creciente clase media. Escolano y Ortiz (2007) asocian la actual aceleración de la suburbanización con tendencias sociales y demográficas. Ellos observan un relativo cambio en el ciclo de vida individual, el cual, por ejemplo, va acompańado de una disminución en el tamaño de los hogares, pasando de 4.1 a 3.7 personas entre 1992 y 2002. Mientras no tengamos constancia de un estudio riguroso sobre preferencias residenciales, se asume que otros factores de preferencia como la creciente demanda de viviendas en un entorno socialmente homogéneo y protegido juegan asimismo un rol importante. Por consiguiente, la suburbanización residencial en Santiago ha sido complementada y corroborada por otra tendencia en Chile y en otros países. La creciente percepción del entorno urbano como inseguro y potencialmente violento ha llevado en las últimas dos décadas a la proliferación de barrios cerrados (Hidalgo et al., 2007). La protección de viviendas, y en general la inseguridad de los espacios públicos y la criminalidad, siempre han sido un tema importante en América Latina. Sin embargo, la demanda por una vivienda segura se ha incrementado, por lo menos en Chile, hacia finales del siglo XX. Ello se ha traducido en una demanda considerable de viviendas en barrios cerrados localizados en la periferia urbana, cuya satisfacción es de alto interés para el capital inmobiliario internacional. 
En relación con el aspecto político de la dispersión, varias políticas y decisiones políticas han facilitado en los últimos años la intensificación del desarrollo de suelo urbano en Santiago. En general, ellas suscriben a la orientación dominante al mercado en Chile, como el bien conocido "delantero" en América Latina en términos de liberalización del mercado y apoyo a la inversión directa. Por otro lado, los instrumentos de planificación de uso del suelo (como los planes reguladores en el nivel local y regional) no parecen ser capaces de contener satisfactoriamente el crecimiento espacial (Petermann, 2006), si es que ellos efectivamente pretenden restringir la dispersión urbana. En muchos casos, los planes de uso de suelo están concebidos más bien para preparar o legitimizar, más que para limitar un nuevo desarrollo. Estas condiciones marco resultan altamente propicias para un modo de producción de viviendas caracterizado por proyectos a gran escala, que normalmente requieren el desarrollo de enormes emplazamientos nuevos en la periferia urbana. Así, afirmamos que las políticas y los desarrollos privados apoyados por las políticas son al menos igualmente importantes que el ciclo de vida y las tendencias de estilo de vida que explican el consumo de suelo suburbano y la dispersión urbana, en un contexto general de crecimiento económico sostenido en Chile.

La distinción entre factores de dispersión relacionados con la demanda y con las políticas es ciertamente instructiva desde una perspectiva analítica. No obstante, tal distinción parece demasiado general e imprecisa cuando se trata de comprender la dinámica de la dispersión. Es evidente que en realidad la dispersión urbana no es ni sólo el resultado predecible de una invariable aspiración humana por una vida suburbana, ni la mera consecuencia de la creación de políticas. Más bien, ambos tipos de fuerzas motrices de la dispersión son interdependientes y se refuerzan entre sí en un marco dado de gobernanza. La reciente historia de la dispersión de Santiago de Chile ofrece una clara explicación de su interrelación. El marketing que el sector inmobiliario realiza para sus viviendas suburbanas y complejos residenciales, que normalmente alude a modelos de estilos de vida extranjeros, p. ej. al norteamericano, induce a una nueva demanda que podría no existir sin la promoción de productos suburbanos. En otras palabras, la promoción de la vivienda suburbana aumenta la demanda de dispersión por parte de los ciudadanos de la misma manera que, por otro lado, los actores públicos y privados se sienten empujados a satisfacer esta demanda, ya sea directamente mediante proyectos residenciales o indirectamente mediante la infraestructura relacionada. Este tipo de refuerzo mutuo de los factores de dispersión existe en casi todos los casos donde el desarrollo suburbano es promovido por las grandes compañías inmobiliarias. Por lo tanto, podríamos sostener que la dispersión urbana en Santiago es, actualmente, una consecuencia, no de la existencia, sino de la interacción de tres fuerzas, que juntas forman el arreglo específico de gobernanza en una situación dada: primero, el suministro y la promoción de viviendas suburbanas por parte del sector inmobiliario; segundo, el apoyo que éstos reciben de las políticas del sector público; y, tercero, los ciudadanos y sus preferencias. Por cierto, esta afirmación requiere de un análisis más riguroso respecto de estas tres fuerzas y su interacción.

\section{Actores, instituciones y procesos decisorios: desenmarańando la dinámica de la disper- sión en Santiago}

La bibliografía existente sobre la aplicación de la gobernanza al análisis de los asuntos urbanos ofrece varios aspectos para entender las fuerzas de la dispersión urbana y su interacción, 
tales como los intereses y las presunciones implícitas de los actores individuales; relaciones, coaliciones y redes formadas por esos actores; así como los patrones y estilos de interacción y barreras de comunicación. Sin embargo, antes de poder aplicar de esa manera el concepto de gobernanza a un "nuevo" caso empírico, debe ser traducido a un diseño apropiado para la investigación. Alain Motte (1996) ha presentado un simple marco categórico a fin de analizar las instancias de la gobernanza urbana: sugiere identificar a los actores relevantes que crean un arreglo concreto de gobernanza y estudiar las relaciones entre estos actores, así como sus referentes cognitivos, representando los últimos el contexto socio-cultural. No obstante, si bien estimamos que esto es un enfoque altamente valioso para cubrir la categoría de "actores" como el primer elemento esencial de gobernanza, como resultado de un extensivo estudio de la bibliografía teórica reciente sobre gobernanza urbana (Nuissl y Heinrichs, 2006) sugeriríamos añadir dos más de esos elementos básicos a las tres categorías de actores (ver Healey, 1996, p. 35f): primero la categoría de las instituciones (en el sentido general de reglas de acción social), y segundo el proceso actual de gobernanza, porque es difícilmente posible deducir su curso y resultados sólo del arreglo comentado de actores e instituciones. Es necesario “(...) poner de relieve los procesos tanto como las organizaciones (...); agencias activas, tanto como estructuras formales; y roles, relaciones y capacidad de movilización (poder de actuar), tanto como el poder sobre recursos y regulaciones" (Healey et al., 2002, p. 15). Por lo demás, el análisis "se centra demasiado en patrones concretos y sobreenfatiza las visibles fuerzas dominantes", mientras que "la dinámica de transformación y los múltiples patrones potenciales que emergen, florecen o son clausurados en procesos de transformación” (Healey et al., 2002, p. 24) continúan ocultos. Por consiguiente, proponemos sistematizar los procesos y la dinámica que al final producirá la dispersión en las tres categorías analíticas: actores, instituciones (políticas), y los propios procesos de toma de decisiones.

La consideración de actores incluye tres aspectos: participación, cultura y relaciones. La participación se refiere a la cuestión según la cual los actores (tanto individuales como colectivos) toman parte activa en procesos específicos de gobernanza urbana, sus intereses, recursos (políticos, financieros, sociales) y, estrechamente relacionados, su respectiva autoridad, competencias y legitimación de la participación. La cultura se refiere al comportamiento, actitudes y rutinas de la propia actuación de los actores involucrados, pero también a la creación social conjunta de presunciones comunes y sensaciones sobre los asuntos contingentes y sus potenciales cursos de acción. Las relaciones se refieren a la multiplicidad de formas y arreglos (en sentido vertical, horizontal o multinivel), según los cuales se organiza la cooperación y la gestión de conflictos.

Las instituciones se refieren a las reglas, tanto formales como informales, que dan forma y condicionan el comportamiento de los actores, así como sus expectativas sobre el comportamiento de los demás. Mientras que las instituciones formales están codificadas (leyes, regulaciones, ordenanzas, planes, etc.), las instituciones informales son normas no escritas y valores, como el agradecimiento por los servicios de un funcionario (p. ej. soborno). Un tercer aspecto que debe ser analizado es la presencia de programas políticos (urbanos) temporales y los subsidios e incentivos relacionados, y su impacto en el comportamiento de los actores. 
Dado que la gobernanza está dirigida hacia problemas concretos y asuntos de desarrollo urbano (en nuestro caso la expansión de la ciudad construida), el proceso de toma de decisiones es un tercer elemento de análisis, que refleja su carácter procedimental y dinámico. En particular, el interés analítico se identifica con cómo los actores del Estado, el sector privado y la sociedad civil consiguen el equilibrio de poder e intereses, así como qué mecanismos de interacción social y coordinación (tanto horizontal como vertical) estructuran los procesos de toma de decisiones. Esto abarca la cuestión de cómo los actores están tratando y compartiendo entre sí los datos y la información, comprendiendo también recomendaciones políticas con base científica, y qué tipo de datos e información se incluyen (o excluyen).

Estas tres categorías y sus inherentes elementos de análisis se aplican más abajo sobre la base de los estudios disponibles, en concreto los de Ducci (2004) y Zunino (2006), quienes investigan las dinámicas constelaciones de actores en vista de proyectos a gran escala (la construcción de la autopista Costanera Norte y el más reciente re-desarrollo del aeropuerto de Cerrillos promovido por el gobierno). Aunque ninguno de los ejemplos corresponde exactamente al asunto de desarrollo de emplazamientos nuevos más allá del área urbana existente, forman una base apropiada para comprender relaciones más generales. A esto añadimos nuestras observaciones iniciales y nos referimos además a otras piezas de la amplia bibliografía disponible sobre Santiago.

\section{Actores}

Tal como fuera argumentado anteriormente, los actores del sector público han creado un marco de políticas que ha redundado en facilitar la dispersión urbana. El nivel nacional de gobierno generalmente le da mucha importancia a Santiago como el centro urbano primario del país (en términos de población, servicios, etc.) y por eso suele promover el crecimiento de la capital. Así, el gobierno central desempeña un importante papel en el desarrollo de infraestructura y la provisión de nuevos desarrollos, en particular a través del Ministerio de Obras Públicas (Ducci, 2004; Orellana, 2007). Sin embargo, la política nacional de vivienda está ampliamente orientada hacia los emplazamientos periféricos, con valores comparativamente mucho menores que el suelo urbano consolidado y, por lo tanto, tal política contribuye considerablemente a la dinámica general de dispersión (Tokman, 2006). Cabe agregar, que las unidades territoriales del gobierno local se caracterizan por su débil base inversora, con las contadas excepciones de las municipalidades de Providencia, Vitacura y Las Condes, que se caracterizan por la homogeneidad de su población residente, situada en los niveles más altos de ingresos, y una importante actividad comercial y de servicios (ver Orellana, 2007). Sin embargo, para la mayoría de las comunas, la única posibilidad de mejorar sus ingresos es mediante las inversiones realizadas en su territorio. Las municipalidades a menudo compiten entre sí para atraer inversiones para el uso urbano de su suelo. Además, la mayoría de municipalidades espera beneficios de planificación, es decir, gastos en infraestructura social por parte de inversionistas privados, a cambio del permiso para desarrollar proyectos inmobiliarios. El gobierno regional, del que pudiera esperarse que desempeñara un importante papel de cara a la regionalización del fenómeno, tiene pocas posibilidades (capacidad y autoridad) para coordinar el desarrollo por y entre un creciente número de comunas que se incorporan a la red funcional megaurbana, pero que se encuentran fuera del actual área de cobertura del "plan regulador intercomunal" 
(Schiappacasse y Müller, 2003). Aunque con la integración de las provincias de Melipilla y Talagante en 2006, todas las municipalidades de la Región Metropolitana de Santiago han sido finalmente incluidas en el Plan Regulador Metropolitano de Santiago (PRMS), este plan sólo contiene prescripciones para el desarrollo de las áreas urbanas. Las áreas rurales siguen, por tanto, "desprotegidas" y expuestas a la presión de los intereses de desarrollo inmobiliario. Además, no está claro si el gobierno regional, en caso de tener más influencia, desarrollaría una actitud crítica hacia la dispersión urbana.

Los actores del sector inmobiliario privado habitualmente se han beneficiado mucho de la orientación general de las políticas chilenas hacia la liberalización y políticas consensuales, y ellos mismos ejercen presión a favor de esa orientación (De Mattos, 2005). Al albor de estas políticas y los correspondientes procesos de reestructuración político-económica a escala global, ha surgido un nuevo tipo de agente inmobiliario que busca la rentabilidad del capital (internacional) y, por consiguiente, explora nuevas oportunidades para valorizar el suelo y desarrollar productos inmobiliarios atractivos (Stockins, 2004; De Mattos, 2007). Las actividades de este agente incluyen selección de emplazamientos, desarrollo del proyecto incluyendo estrategias de comercialización y financiación, y finalmente la ejecución de la obra. Parte importante de este tipo de desarrollo inmobiliario es realizado en forma de megaproyectos de gran escala en la periferia urbana. (Borsdorf e Hidalgo, 2005a y 2005b). El hecho de que los grandes inversionistas inmobiliarios en Chile tradicionalmente tengan fuertes lazos con las élites económicas del país, las "grandes familias económicas", ha facilitado las actividades del sector privado en el mercado inmobiliario y del suelo. Además, ellos tienen un fuerte poder organizativo y político: la Cámara Chilena de Construcción y la Asociación de Desarrolladores Inmobiliarios se encuentran entre los grupos de presión económica más influyentes del país.

En la actualidad la sociedad (civil) juega un rol importante en la dinámica de dispersión urbana. En efecto, ella ocasiona el enorme número de familias que se muda a una vivienda en un emplazamiento de nuevo desarrollo. Es importante señalar que, como se argumentara más arriba, esta contribución de la sociedad a la dinámica general de dispersión no es necesariamente una expresión de los deseos humanos universales, pues puede afirmarse casi de la misma manera que radica en el aliciente de la orientación del estilo de vida suburbano, p. ej. instituciones informales favorables a los suburbios (ver abajo), por medio de marketing y promoción. Por otro lado, la fuerte coalición del sector público y privado en favor de la dispersión de las zonas residenciales apenas encuentra oposición. Normalmente no hay formación de coaliciones de no crecimiento reales, lo cual es un fenómeno frecuente en los EEUU o Europa (ver Zunino, 2006). A menudo hay una simple razón para esta falta de interés ciudadano: en muchos casos, y en concreto respecto de los megaproyectos en emplazamientos periurbanos, los nuevos desarrollos se producen en espacios en gran parte vacíos y, por ende, afecta sólo a un número relativamente pequeño de población. Con este trasfondo es muy difícil generar participación local. No obstante, hay una razón más profunda que explica la ausencia de la sociedad civil de la dinámica de dispersión y es la fuerte asociación entre los sectores público y privado que se ha desarrollado en Chile antes y después de 1990. Esto frecuentemente conlleva a un enmarcado político de proyectos específicos fuertemente respaldado por los sectores más influyentes en la ciudad, que desalienta la participación. Por otra parte, a menudo es muy difícil para la población afectada por un determinado proyecto de desarrollo obtener suficiente información sobre lo 
que está pasando, y menos aún información sobre sus derechos democráticos en relación con su participación o intervención en el proceso planificador.

Por cierto, es habitual que exista un severo desequilibrio de poder entre los ciudadanos perjudicados por los nuevos desarrollos inmobiliarios y los actores influyentes interesados en los megaproyectos, como agencias inmobiliarias, el sector público y los ciudadanos más ricos, hacia quienes se dirigen normalmente estos desarrollos. Mientras que los agentes de los sectores privados y gubernamentales (en especial del nivel nacional) parecen trabajar en equipo, la voz de una ciudadanía organizada está en gran parte ausente. No obstante, en los últimos años ha habido ejemplos de resistencia ciudadana que han tenido éxito contra proyectos de desarrollo y urbanización (Parraguez Sánchez, 2008). Estos ejemplos muestran que también bajo las condiciones actuales es posible conseguir la participación de la sociedad civil. Aparentemente tal participación requiere dos precondiciones: primero, un número suficiente de personas afectadas directamente por un proyecto (p. ej., porque la realización de éste puede transformar completamente su entorno cotidiano) y segundo, las personas necesitan un fuerte sentimiento de identidad y pertenencia a un vecindario (lo cual de ninguna manera es siempre el caso en Santiago). El tipo de participación ciudadana que a menudo se desarrolla cuando se dan estas condiciones, no obstante, parece formar una base sobre la cual pueden evolucionar formas de ciudadanía más ambiciosas y de mayor alcance (Parraguez Sánchez, 2008). Además, nuestras investigaciones nos proporcionan algunas evidencias preliminares sobre organizaciones de la sociedad civil que surgieron ligadas a un territorio en particular, o bien, a modo de $N I M B Y$ 's, que cada vez se involucran más en discusiones sobre el marco normativo general del desarrollo urbano. Un buen ejemplo es la actual modificación de la Ley General de Urbanismo y Construcción, que recogerá importantes novedades normativas sobre planificación condicionada y megaproyectos periurbanos. En el transcurso de este proceso legislativo, las organizaciones de la sociedad civil han presentado al respecto cada vez más declaraciones y objeciones, demostrando así una ampliación de su ámbito de acción.

\section{Instituciones}

El apoyo antes mencionado a la dispersión urbana en la política chilena se refleja en varias leyes y reglamentos que han sido aprobados en Chile en los últimos años. En 1979, por ejemplo, se eliminó el así llamado límite urbano, una poderosa restricción (al menos en términos legales) al desarrollo de emplazamientos nuevos. Al mismo tiempo se aprobó una ley que sustituía en parte a la ley de límite urbano. Ella prohibió la subdivisión de parcelas por debajo de los $5000 \mathrm{~m}^{2}$ en áreas rurales, de manera de preservar el paisaje rural. Sin embargo, respecto de las llamadas parcelas de agrado en los alrededores periurbanos de Santiago, esta ley tiene hoy el efecto contrario. Ya no previene el uso exclusivamente residencial de emplazamientos rurales. Más bien ha provocado un peculiar tipo de dispersión urbana, caracterizado por la adquisición y preparación para la urbanización de tales emplazamientos por parte de desarrolladores inmobiliarios, que luego los venden al creciente número de hogares adinerados que pueden permitirse residir en tan grandes parcelas (Naranjo, 2006).

A pesar de que en 1985 se deshizo la eliminación del limite urbano y aunque el Plan Regulador Metropolitano de Santiago de 1994 volviera a definir límites urbanos, no se pudo evitar que las 
áreas residenciales se derramaran por la campiña periurbana. Esta debilidad se debe en parte a la incorporación de varias comunas que se encontraban más allá del área construida de Santiago de Chile al área de expansión de la ciudad, mediante desarrollos inmobiliarios a gran escala. La inclusión de estos megaproyectos residenciales se hizo posible gracias a nuevas regulaciones aprobadas entre 1997 y 2004, como la Zonas de Urbanización Condicionada (ZODUC), Áreas de Urbanización Prioritaria (ADUP) y Proyectos de Desarrollo Urbano Condicionado (PDUC), que permitieron el desarrollo residencial más allá de los límites de la ciudad (Hidalgo, 2005). En el debate actual existe una evaluación mixta de estos instrumentos, tanto en los medios de comunicación como en la comunidad científica. Mientras que para algunos aquéllos suponen claramente una nueva forma positiva de construir ciudades (Poduje 2006), otros los ven más bien como meros medios para legitimizar la continuación irrestricta de la dispersión urbana.

El abanico de instituciones formales que se han mostrado propicias a la dispersión urbana van en la misma dirección que un amplio espectro de instituciones informales, donde destaca la misma visión general de vivienda digna y entornos residenciales atractivos que predominan en la sociedad chilena y que constituye el fundamento sociocultural de la demanda de dispersión residencial. Es más, esta visión de las instituciones informales es reforzada por las formales. Por ejemplo, el apoyo a agencias inmobiliarias que desarrollan áreas residenciales suburbanas (la mayoría cerradas), a través de la Ley General de Urbanismo y Construcción y otros documentos de planificación, ayuda sin duda a expandir una orientación suburbana. Tal influencia de las instituciones formales sobre las informales es, por supuesto, una común, y normalmente bienvenida, característica de las sociedades humanas. Es sorprendente, sin embargo, que en el caso de la dispersión urbana esta influencia exista principalmente en un modo indirecto, en que las instituciones formales ayudan a desarrollar emplazamientos suburbanos, cuya oferta y comercialización provoca un nuevo entendimiento común de un estilo de vida decente.

Sin embargo, no son sólo las instituciones formales las que influyen en el desarrollo y cambio de las instituciones informales. En lo que respecta a dispersión urbana, las actividades del sector privado pueden ejercer tal influencia incluso más allá del marco (legal) de las instituciones formales. En Santiago, esto es especialmente obvio con el vibrante desarrollo de las urbanizaciones cerradas que en la actualidad se está expandiendo por todas partes. Parece ser que lo que impulsa esta tendencia en primer lugar es la oferta y comercialización de los barrios cerrados por parte del sector inmobiliario, más que la percepción de un entorno inseguro. En la actualidad es casi imposible conseguir una nueva casa o departamento que no esté en una urbanización cerrada. La campańa sistemática de vida cerrada realizada por el sector inmobiliario contribuye a los actuales discursos sobre seguridad y calidad de vida y ha hecho que vivir en un entorno vigilado se convierta en un símbolo de estatus que antes no existía. En la actualidad las personas quieren vivir en urbanizaciones cerradas para diferenciarse de los grupos sociales más bajos. Más que la inseguridad en términos de violencia, las personas en una sociedad desigual como la chilena suelen sentir inseguridad en su estatus social. Vivir en una comunidad cerrada parece un modo apropiado de superar este miedo y de asegurarse que uno no es confundido con grupos sociales más bajos.

Podemos concluir que la base institucional de dispersión urbana es bastante fuerte en Santiago, lo cual planteará un problema especial si las políticas futuras pretenden contener la dispersión. 


\section{Toma de decisiones}

Los procesos de toma de decisiones que resultan en el desarrollo del suelo en la periferia urbana corresponden probablemente a la más difícil de las tres categorías analíticas propuestas, ya que su análisis requiere un detallado estudio empírico de procesos políticos. Además, varios trabajos realizados por distintos autores sobre el reciente desarrollo urbano de Santiago resaltan a esta ciudad como un tipo de modelo de las metrópolis globalizadas de América Latina. Ellos subrayan que el modo en que tiene lugar la dispersión urbana en Santiago sólo puede ser entendido con el trasfondo de las características más fundamentales de la política y gobernanza chilenas (e.g., De Mattos, 1999; Dockemdorff et al., 2000; Fuentes y Sierralta, 2004). Entre estas características se encuentra la aún considerable concentración de poder gubernamental en el nivel nacional. En el curso de las políticas de descentralización, no obstante, actualmente podemos observar una tendencia hacia la transferencia de al menos algunas responsabilidades funcionales hacia el nivel local. Esta tendencia transforma, potencialmente, a los gobiernos locales en actores importantes. Sin embargo, la alta dependencia de los municipios de las asignaciones económicas del Estado, sobre todo en el caso de aquéllos que tienen altos porcentajes de población pobre, limita la acción posible de estos actores. Normalmente, los municipios usarán su derecho de asignar derechos de uso del suelo, ofreciendo suelo fresco a potenciales inversionistas y desarrolladores inmobiliarios, apoyando de este modo la política del gobierno nacional de estimular la inversión directa. Esta situación normalmente facilita que se organice el consentimiento político para un proyecto de desarrollo en particular. El inconveniente de estas estructuras de toma de decisiones, sin embargo, son tanto la enorme influencia del sector privado en el negocio de construir ciudad, así como la falta de oportunidades para que la población local participe en los procesos de desarrollo urbano.

\section{Nuevos desafíos para la gobernanza metropolitana}

A partir de lo expuesto en el presente artículo, concluimos que lo que en la introducción definimos como régimen de dispersión urbana en Santiago de Chile, puede ser atribuido -por un lado- a diferentes características comunes a la mayoría de las ciudades de mayor tamaño de América Latina, mientras que -por otro lado- algunas dimensiones del fenómeno sólo pueden ser entendidas en el contexto específico del caso. Entre los factores comunes están el cambio demográfico y nuevas preferencias para la elección de emplazamiento de vivienda, una intensificación de la inversión en grandes proyectos de desarrollo residencial y la relajación del control y de las políticas restrictivas, en un marco general pro-crecimiento. Otro aspecto importante que concierne a la mayoría de las megaciudades de América Latina, pero que es especialmente pronunciado en Santiago, se refiere a las responsabilidades específicas y relaciones de actores, entre los distintos niveles de gobierno (Orellana, 2007). La comprensión de la lógica del desarrollo urbano, desde una perspectiva local, en el ejemplo de Santiago, ha de considerar, por un lado, el rol aún bastante fuerte del gobierno nacional; en tanto por otro, destaca -en el mejor de los casos- la descoordinación de las actividades de un número significativo de municipios independientes, aunque a menudo tales actividades se manifiestan competitivas entre sí, entre municipalidades que rivalizan por la atracción de inversiones y otros recursos. Por cierto, es una tarea desafiante para la gobernanza metropolitana en Santiago de Chile desarrollar mecanismos que traten con la metrópolis en expansión, abordando ambos tipos de factores. 
Para cumplir esta tarea, en vez de abogar a favor de instrumentos reguladores más estrictos (cuya implementación exitosa parece dudosa), preferimos aconsejar investigar el potencial de un conjunto de otras medidas, que en general refuercen las políticas de descentralización en un marco adecuado de coordinación, así como el desarrollo de instrumentos que operen más bajo la aplicación de incentivos, que bajo medidas coercitivas o restrictivas.

Primero, es claro que las políticas de dispersión urbana sólo pueden tener éxito si se solucionan los problemas de gobernanza multinivel. Aunque el gobierno chileno ha realizado una apuesta fuerte en el desarrollo de una política urbana, las actividades de los diferentes ministerios nacionales que inciden en su puesta en práctica están escasamente coordinadas. Esta falta de coordinación horizontal a nivel nacional se agrava por la falta de coordinación vertical entre los niveles de gobierno central, regional y local. Por último, aunque no menos importante, la falta de coordinación horizontal a nivel local se complica por la duplicidad de muchas actividades desarrolladas simultáneamente por distintas municipalidades, las que compiten entre sí, en lugar de sincronizar sus actividades. Una estrategia para superar la actual situación de acciones descoordinadas y a menudo contradictorias de las autoridades, que escasamente integran perspectivas regionales y municipales sobre desarrollo metropolitano, puede ser la introducción de metas y objetivos comunes sobre consumo de suelo, así como incentivos para cooperación intercomunal (voluntaria). Tales incentivos pueden ser provistos, por ejemplo, por los llamados instrumentos económicos de política de uso del suelo, como los presupuestos de suelo, certificados de suelo transferible o fondos de redistribución intercomunal. La aplicación de estos incentivos a la realidad de Santiago, mediante reglamentaciones adecuadas que eviten el mal uso de estos instrumentos (como por ejemplo la transferencia de suelos de distinta calidad y aptitud de uso), es un tema que requiere de estudios específicos.

Segundo, y estrechamente relacionado con el problema de la falta de coordinación, hay un desequilibrio de poder e influencia entre los diferentes niveles de gobierno. En la actualidad, el desarrollo urbano en Chile, y en particular en la capital, está dominado en su mayoría por políticas gubernamentales de nivel nacional, mientras que las instituciones de nivel regional dependen de estos ministerios y sólo tienen débil poder. La máxima autoridad del gobierno regional (intendente) no es elegida públicamente, sino que es designada por el Presidente de Chile, por consiguiente difícilmente puede representar a la región y a su gente. Por cierto, las posibilidades de la sociedad civil de participar activamente en las negociaciones sobre proyectos concretos aumentaría, y entonces también su involucramiento en el desarrollo de la región urbana en su conjunto, si el nivel regional de gobierno estuviera legitimado en un proceso democrático. Además, esto probablemente estimularía la coordinación y también minimizaría la competencia entre la unidades del gobierno local.

Tercero, se requeriría información pertinente para advertir al gobierno en todos sus niveles sobre las implicaciones de la continua expansión urbana. Esto incluye, por ejemplo, la predicción de escenarios sobre la localización de demanda futura de viviendas y la estimación de los costos futuros asociados (en particular relacionados con la infraestructura o el consumo de energía) y degradación medioambiental. Esto podría estar complementado por la evaluación de los potenciales de reserva de la ciudad interior (en particular los sitios eriazos). 
Cuarto, pensamos que es necesario un discurso público informado e intenso sobre la dispersión, antes de que pueda producirse algún potencial para cambiar de forma fundamental la hasta ahora fuerte base institucional (formal e informal) hacia la dispersión urbana. Tal discurso podría estar vinculado con una discusión pública, que incluya una fuerte participación de la sociedad civil sobre el futuro deseado para la Región Metropolitana, así como sobre la estrategia para conseguir este objetivo.

\section{Referencias bibliográficas}

Arriagada C. \& Simioni, D. (2001). Dinámica de valorización de suelo en el área metropolitana del Gran Santiago y desafios de financiamiento urbano. Santiago de Chile: ECLAC/CEPAL, Serie Medio Ambiente y Desarrollo.

Bähr, J. \& Mertins, G. (1995). Die lateinamerikanische Großstadt. Verstädterungprozesse und Stadtstrukturen. Darmstadt: Wissenschaftliche Buchgesellschaft.

Borsdorf, A. \& Hidalgo, R. (2005a). Los Mega-Diseños residenciales valladores en las periferias de las Metrópolis Latinoamericanas y el advenimiento de un nuevo concepto de ciudad. Alcances en base al caso de Santiago de Chile. Scripta Nova, Revista Electrónica de Geografía y Ciencias Sociales 9, 194, 3.

Borsdorf, A. \& Hidalgo, R. (2005b). Städtebauliche Megaprojekte im Umland lateinamerikanischer Metropolen. Eine Antithese zur Stadt? Geographische Rundschau, 57, 10, 30-38.

De Lázaro y Torres, M.L., González, M.J. \& Izquierdo, S. (2003). Reflexiones sobre la terminología para designar a las ciudades que encabezan la jerarquía mundial. En La ciudad: nuevos procesos, nuevas respuestas (pp. 63-74), Actas del VI Coloquio de Geografía Urbana, Universidad de León, León, edición digital.

De Mattos, C. \& Hidalgo, R. (Eds.) (2007). Santiago de Chile: movilidad espacial y reconfguración metropolitana. Santiago: Serie GEOlibros 8, Instituto de Estudios Urbanos y Territoriales, Pontificia Universidad Católica de Chile.

De Mattos, C. A. (1999). Santiago de Chile, globalización y expansión metropolitana: lo que existía sigue existiendo. EURE, 25, 76, 29-56.

De Mattos, C. A. (2005). Santiago de Chile: modernización capitalista y transformación metropolitana. En M. Carmona (Ed.), Globalización y grandes proyectos urbanos. La respuesta de 25 ciudades. Buenos Aires: Ediciones Infinito.

De Mattos, C. A. (2007). Globalización, negocios inmobiliarios y transformación urbana. Nueva Sociedad 212, Noviembre/Diciembre 2007. Rescatado el 5 de agosto de 2008 de www.nuso.org/upload/articulos/3481_1.pdf.

De Ramón (2007). Santiago de Chile (1541-1991). La historia de una sociedad urbana. Santiago: Catalonia.

Dockemdorff, E., Rodríguez, A. \& Winchester, L. (2000). Santiago de Chile: metropolization, globalization and inequity. Environment and Urbanization 12, 1, 171-183.

Ducci, M. E. \& González, M. (2006). Anatomía de la expansión de Santiago. En Galetovic, A. (Ed.), Santiago: dónde estamos y hacia dónde vamos (pp. 123-146). Santiago: Centro de Estudios Públicos.

Ducci, M. E. (2000). Santiago: territorios, anhelos y temores. Efectos sociales y espaciales de la expansión urbana. EURE 26, 79, 5-24. 
Ducci, M. E. (2004). Las batallas urbanas de principios del tercer milenio. Urbared. Recuperado el 2 de febrero de 2007 de www.urbared.ungs.edu.ar/recursos_bibliografia_trabajos_consul.htm.

Escolano, S. \& Ortiz, J. (2007). Patrones espaciales de movilidad de la población: algunos efectos en la sociogeografía del gran Santiago. En C. De Mattos \& R. Hidalgo (Eds.), Santiago de Chile: movilidad espacial y reconfiguración metropolitana (pp. 53-66). Santiago: Serie GEOlibros 8, Instituto de Estudios Urbanos y Territoriales, Pontificia Universidad Católica de Chile.

Fuentes, L. \& Sierralta, C. (2004). Santiago de Chile, ¡ejemplo de una reestructuración capitalista global? EURE 30, 91, 7-28.

Galetovic, A. \& Jordán, P. (2006). Santiago: dónde estamos. Hacia dónde vamos. En A. Galetovic (Ed.), Santiago: dónde estamos y hacia dónde vamos (pp. 25-72). Santiago: Centro de Estudios Públicos.

Galetovic, A. (Ed.) (2006). Santiago: dónde estamos y hacia dónde vamos. Santiago: Centro de Estudios Públicos.

Green, M. \& Soler, F. (2005). Santiago: de un proceso acelerado de crecimiento a uno de transformaciones. En C. De Mattos, M. E. Ducci, A. Rodríguez \& G. Yáñez (Eds.) Santiago en la globalización, ¿una nueva ciudad? (pp. 47-84). Santiago: Colección EURE Libros/Ediciones SUR.

Healey P. (1996). An institutionalist approach to spatial planning. En P. Healey, A. Khakee, A. Motte \& B. Needham (Eds.) Making strategic spatial plans: innovation in Europe (pp. 21-36). London: UCL Press.

Healey, P., Cars, G., Madanipour, A., De Magelhaes, C. (2002). Transforming governance, insitutionalist analysis and institutional capacity. En G. Cars, P. Healey, A. Madanipour, C. De Magelhaes (Eds.) Urban governance, institutional capacity and social milieux (pp. 6- 28). Aldershot: Ashgate.

Hidalgo, R. (2005). Post-suburbia ou post-urbia? Les mégaprojets résidentiels dans la périphérie de Santiago du Chili. Revue Géographique de l'Est, 45, 3-4, 209-217.

Hidalgo, R., Borsdorf, A. \& Sánchez, R. (2007). La expansión residencial amurallada en la reconfiguración metropolitana de Santiago de Chile. En C. De Mattos \& R. Hidalgo (Eds.), Santiago de Chile: movilidad espacial y reconfiguración metropolitana (pp. 117-136). Santiago: Serie GEOlibros 8, Instituto de Estudios Urbanos y Territoriales, Pontificia Universidad Católica de Chile.

Instituto de Estudios Urbanos de la Pontificia Universidad Católica de Chile, IEUT (2003). Perspectivas del medio ambiente urbano: GEO Santiago de Chile. Santiago de Chile: IEUT.

Jackson, K. T. (1985). Crabgrass frontier. The suburbanization of the United States. New York/ Oxford: Oxford University Press.

Lopez, E. J. (2006). Impacto del crecimiento del Gran Santiago en el deterioro funcional de sus espacios pericentrales. En H. Capel \& R. Hidalgo (Eds.), Construyendo la ciudad del siglo XXI. Retos y perspectivas urbanas en España y Chile (pp. 337-350). Santiago de Chile: Serie GEOlibros 6, Instituto de Geografía, Pontificia Universidad Católica de Chile.

Motte, A. (1996). The institutional relations of plan-making. En P. Healey, A. Khakee, A. Motte \& B. Needham (Eds.) Making strategic spatial plans: innovation in Europe (pp. 231-254). London: UCL Press. 
Naranjo, G. (2006). Efectos del Plan Regulador Metropolitano de Santiago en el ordenamiento de los espacios periurbanos. En H. Capel \& R. Hidalgo (Eds.), Construyendo la ciudad del siglo XXI. Retos y perspectivas urbanas en España y Chile (pp. 293-306). Santiago de Chile: Serie GEOlibros 6, Instituto de Geografía, Pontificia Universidad Católica de Chile.

Nuissl, H. \& Heinrichs, D. (2006). Zwischen Paradigma und heißer Luft: Der Begriff der Governance als Anregung für die räumliche Planung. En U. Altrock, S. Güntner, S. Huning, T. Kuder, H. Nuissl \& H. Peters (Eds.), Sparsamer Staat-Schwache Stadt? (pp. 51-72). Berlin: Planungsrundschau 13, Verlag Uwe Altrock.

Orellana, A. (2005). El control social del espacio metropolitano desde lo local. En C. De Mattos, O. Figueroa, A. Orellana \& G. Yánez (Eds.), Gobernanza, competitividad y redes: la gestión en las ciudades del siglo XXI (pp. 263-296). Santiago: Colección EURE libros.

Orellana, A. (2007). La gobernabilidad metropolitana: nuevos escenarios para el desarrollo urbano y territorial del área metropolitana de Santiago. En C. De Mattos \& R. Hidalgo (Eds.), Santiago de Chile: movilidad espacial y reconfiguración metropolitana (pp. 189206). Santiago: GEOlibros 8, Instituto de Estudios Urbanos y Territoriales, Pontificia Universidad Católica de Chile.

Parraguez Sánchez, L. (2008). Emergent social movements in Santiago. Between the defense of spatial identity and the fight for city rights. The case of the residents assembly of José Maria Caro. Pontificia Universidad Católica de Chile, Master Thesis.

Paz Castro, C. (2006). Impacto de la dispersión urbana de la ciudad de Santiago en la calidad del suelo en la periferia norte: Colina y Lampa. En H. Capel \& R. Hidalgo (Eds.), Construyendo la ciudad del siglo XXI. Retos y perspectivas urbanas en España y Chile (pp. 279-291). Santiago de Chile: Serie GEOlibros 6, Instituto de Geografía, Pontificia Universidad Católica de Chile.

Peiser, R. (2001). Decomposing urban sprawl. Town Planning Review 72, 3, 275-298.

Petermann, A. (2006). ¿Quién extendió a Santiago? Una breve historia del límite urbano 19532004. En A. Galetovic (Ed.) Santiago: dónde estamos y hacia dónde vamos (pp. 205-230). Santiago: Centro de Estudios Públicos.

Poduje, I. (2006). El globo y el acordeón: planificación urbana en Santiago, 1960-2004. En A. Galetovic (Ed.), Santiago: dónde estamos y hacia dónde vamos (pp. 231-276). Santiago: Centro de Estudios Públicos.

Rodríguez, J. (2007). Paradojas y contrapuntos de dinámica demográfica metropolitana: algunas respuestas basadas en la explotación intensiva de microdatos censales. En C. De Mattos \& R. Hidalgo (Eds.), Santiago de Chile: movilidad espacial y reconfiguración metropolitana (pp. 19-52). Santiago: Serie GEOlibros 8, Instituto de Estudios Urbanos y Territoriales, Pontificia Universidad Católica de Chile.

Romero, H. \& Vázquez, A. (2006). La comodificación de los territorios urbanizables y la degradación ambiental en Santiago de Chile. En H. Capel \& R. Hidalgo (Eds.), Construyendo la ciudad del siglo XXI. Retos y perspectivas urbanas en España y Chile (pp. 263-278). Santiago: Serie GEOlibros 6, Instituto de Geografía, Pontificia Universidad Católica de Chile.

Schiappacasse, P. \& Müller, B. (2003). Desarrollo metropolitano integrado: el caso de Santiago de Chile. Urbano 7, 10, 68-74.

Smith, N. (1979). Toward a theory of gentrification: a back to the city movement by capital, not people. Journal of the American Planning Association 45, 4, 538-548. 
Stockins, P. (2004). Oferta y demanda de vivienda en la periferia santiaguina: los nuevos desarrollos inmobiliarios. En G. Cáceres \& F. Sabatini (Eds.), Barrios cerrados en Santiago de Chile. Entre la exclusión y la integración residencial. Santiago: Ed. Lincoln Institute, Pontificia Universidad Católica de Chile.

Tokman, A. (2006). El MINVU, la política habitacional y la expansión excesiva de Santiago. En A. Galetovic (Ed.), Santiago: dónde estamos y hacia dónde vamos (pp. 489-522). Santiago: Centro de Estudios Públicos.

Zunino, H. M. (2006). Power relations in urban decision-making: neo-liberalism, 'technopoliticians' and autoritarian redevelopment in Santiago, Chile. Urban Studies 43, 10, 1825-1846. 